\title{
Endoscopically guided bladder neck suspension for continence in paraplegic women with implant driven micturition
}

\author{
L Cardozo MD FRCOG, G S Brindley MD FRCP HonFRCS FRS
}

\author{
King's College Hospital, London SE5 $9 R S$ and MRC Neurological Prostheses Unit, \\ Institute of Psychiatry, London SE5 8AF, England.
}

Two paraplegic women who had undergone implantation of a sacral anterior nerve root stimulator to treat their voiding difficulties and recurrent urinary tract infections subsequently suffered from stress incontinence. After urodynamic evaluation they were treated with a Stamey operation which rendered them dry without hindering their implant driven micturition.

Key words: paraplegia; implant; stress incontinence; Stamey procedure.

\section{Introduction}

Most women with spinal cord injuries suffer from reflex urinary incontinence. In many of them, this persists despite intermittent self catheterisation and anticholinergic drugs. It can, however, almost always be abolished by sacral posterior rhizotomy and implantation of a sacral anterior root stimulator. ${ }^{1-5}$ This surgical treatment has collateral advantages in eliminating urinary tract infection, ${ }^{2-5}$ improving bladder compliance and thus avoiding back pressure damage to the upper urinary tracts, ${ }^{2-5}$ abolishing high pressure ureteric reflux $^{2}$ and improving colorectal function. ${ }^{6}$

Cure of reflex incontinence by sacral posterior rhizotomy sometimes unmasks previously unsuspected genuine stress incontinence due to urethral sphincter incompetence. It may perhaps sometimes create genuine stress incontinence that did not exist previously, by diminishing reflex tone in the pelvic floor muscles or by accidental damage to some sacral anterior roots, though there is no evidence that decrease in the tone of the pelvic floor is a regular consequence of sacral posterior rhizotomy.

We here report the management of 2 women who had sacral anterior root stimulators implanted, with sacral posterior rhizotomy, in 1985. They were then free from reflex incontinence and emptied their bladders well by means of their implants, but they continued to suffer from stress incontinence until this was treated.

\section{Case I}

A female patient of 64 years sustained a sensory incomplete motor almost complete spinal cord lesion at C6 in an accident in 1972. She had had an indwelling catheter from 1973 to 1977 but after removal of a bladder stone in 1977 , she ceased to use a catheter and was severely incontinent. Urodynamics in June 1985 revealed severe detrusor hyperreflexia with a bladder capacity of $100 \mathrm{ml}$, systolic detrusor contractions up to $42 \mathrm{~cm}$ of water and reflex voiding. There was mild trabeculation and one small diverticulum. No vesicoureteric reflux occurred during filling or involuntary reflex voiding. The bladder neck was open at rest. She was unable to void voluntarily. In July 1985 a sacral anterior nerve root stimulator was implanted. Following this the patient was able to void using her implant. She still complained of incontinence, and in January 1986 urodynamic studies revealed a stable bladder with capacity of $400 \mathrm{ml}$. The bladder neck was open at rest. There was leakage of contrast medium down the urethra from $400 \mathrm{ml}$ onwards in the absence of abnormal detrusor activity. Implant driven micturition was highly effective, emptying the bladder to completion with one burst. There was no evidence of trabeculation, diverticula or vesicoureteric reflux.

The incontinence continued to be a problem requiring the use of large incontinence pads and causing excoriation of the vulva. In 
view of the fact that leakage occurred on movement because of raised intraabdominal pressure and not a rise in detrusor pressure, a diagnosis of urethral sphincter incompetence was made and in September 1986 a Stamey operation was undertaken under general anaesthesia. A suprapubic catheter was inserted at the end of the operation. Postoperatively the patient made an uncomplicated recovery and 6 days postoperatively the catheter was clamped and she resumed implant driven micturition. Within one week she was emptying her bladder efficiently with residual urines of less than $50 \mathrm{ml}$. Unfortunately she developed a Pseudomonas urinary tract infection which required intravenous antibiotic therapy. In total she was admitted to hospital for 5 weeks. Thereafter she remained dry and was able to void efficiently using her implant at 6 to 8 hour intervals. In February 1987 she wrote to say that her operation had been completely successful. She was last reviewed in March 1991, when she was $100 \%$ continent, voided by implant with a residual less than $20 \mathrm{ml}$, and had not had a further urinary tract infection.

\section{Case II}

A female patient of 33 years sustained a T5 complete injury in a road traffic accident in 1982. Thereafter she voided by percussion leaving large urinary residuals, suffered from recurrent urinary tract infections and was severely incontinent. Urodynamics in May 1984 revealed detrusor hyperreflexia with a urinary residual in excess of $100 \mathrm{ml}$, a bladder capacity of $220 \mathrm{ml}$ and detrusor contractions up to $46 \mathrm{~cm}$ of water associated with leakage of contrast per urethram. Voiding was achieved in bursts by suprapubic percussion leaving a large urinary residual. The bladder was slightly trabeculated with no diverticula and no vesicoureteric reflux. A sacral anterior nerve root stimulator was implanted in June 1985, and thereafter micturition was implant driven. Postoperative urodynamic assessment revealed a bladder capacity of $450 \mathrm{ml}$. There were no systolic reflex contractions, detrusor compliance was normal $\left(14 \mathrm{~cm} \mathrm{H}_{2} \mathrm{O}\right.$ pressure rise on filling to $450 \mathrm{ml}$ ), and the bladder could be completely emptied using the stimulator. However, there was severe incontinence evident on increased intraabdominal pressure and urethral pressure profilometry revealed a maximum urethral closure pressure of $4 \mathrm{~cm}$ of water. From September 1985 the patient was treated with desipramine $25 \mathrm{mg}$ t.d.s. This greatly diminished her incontinence, but she continued to have occasional leaks.

Therefore in 1989 a Stamey procedure was undertaken. The operation was uncomplicated and she was able to void using her implant 5 days postoperatively and was discharged from hospital 7 days postoperatively. When she was seen in the outpatients clinic 6 weeks later, she was dry but complained of frequency of micturition. She was treated for urinary tract infection and the frequency of micturition resolved. Follow up urodynamic studies carried out later that year revealed a bladder capacity of $550 \mathrm{ml}$ with minimal abnormal detrusor activity and no leakage of contrast medium per urethram. Implant driven micturition resulted in voiding to completion. There was no trabeculation, diverticula or vesicoureteric reflux. Unfortunately, rapid desipramine withdrawal caused depression and tricyclic antidepressant therapy had to be reinstituted and withdrawn slowly. Her last follow up was in February 1991, when she reported that she was never incontinent, could void efficiently and had had no urinary infections for over a year. She volunteered that she was so confident about being dry that she had bought leather trousers!

\section{Discussion}

A Stamey procedure ${ }^{7}$ is an endoscopically guided bladder neck suspension which is quick and easy to perform and carries a low morbidity. Although few objective comparative studies have been performed, its success at curing genuine stress incontinence in fit, healthy women is lower than that of a colposuspension. ${ }^{8}$ This is probably due to the fact that the bladder neck is maintained in an intraabdominal position by two nylon sutures in the Stamey, rather than by fibrosis in the colposuspension. However, 
this may not be important in women who are wheelchair bound. For the Stamey operation 2 small incisions are made in the vaginal epithelium on either side at the level of the bladder neck and 2 number 2 nylon sutures are inserted retropubically from the vagina to emerge suprapubically. Silastic buffers are threaded over the nylon on both the vaginal side and the abdominal side and rest subepithelially in the vagina and over the rectus sheath suprapubically. Positioning of the sutures is checked using a cystoscope, the vaginal epithelium is closed and the sutures tied under just sufficient tension to prevent urinary leakage when the bladder is full and suprapubic pressure is exerted. The skin incisions are closed and a suprapubic catheter is inserted.

Genuine stress incontinence is a condition caused by weakness or incompetence of the urethral sphincter mechanism and affects about $60 \%$ of women who complain of urinary leakage. It is commonly treated surgically in healthy women, but in view of the voiding difficulties which patients with spinal injuries usually encounter, surgical treatment is not normally considered a realistic option for such patients. However, the 2 women described in this report have both had sacral anterior nerve root stimula- tors implanted to enable them to void efficiently and the relative outflow obstruction and repositioning caused by the Stamey procedure did not prevent their implants from working efficiently. Thus they were cured of their voiding difficulties and consequently of their recurrent urinary tract infections by the implant and subsequently cured of their stress incontinence by the Stamney procedure. These 2 operations vastly improved the quality of life of our 2 patients, and also reduced their likely morbidity from excoriation of the skin and consequent pressure sores and from the risk of upper urinary tract damage from voiding difficulties and recurrent urinary tract infections.

\section{Conclusions}

Quality of life is just as important for paraplegics as for healthy members of the population and incontinence is a most degrading and socially unacceptable problem which is not usually life threatening and therefore is often ignored. In the light of this report we feel that urethral sphincter incompetence in paraplegics with implant driven micturition can be easily and safely cured using a simple bladder neck suspension.

\section{References}

1 Brindley GS, Polkey CE, Rushton DN (1982) Sacral anterior root stimulators for bladder control in paraplegia. Paraplegia 20: 365-381.

2 Brindley GS, Polkey CE, Rushton DN, Cardozo L (1986) Sacral anterior root stimulators for bladder control in paraplegia: the first 50 cases. J Neurol Neurosurg Psychiatry 49: 1104-1114.

3 Sauerwein D (1990) Die operative Behandlung der spastischen Blasenlähmung bei Querschnittlähmung. Urologe (A) 29: 196-203.

4 Brindley GS, Rushton DN (1990) Long-term follow-up of patients with sacral anterior root stimulator implants. Paraplegia 28: 469-475.

5 MacDonagh RP, Forster DMC, Thomas DG (1990) Urinary continence in spinal injury patients following complete sacral posterior rhizotomy. Brit J Urol 66: 618-622.

6 MacDonagh RP, Sun WM, Smallwood R, Forster DMC, Read NW (1990) Control of defaecation in patients with spinal injuries by stimulation of sacral anterior nerve roots. $\mathrm{Br}$ Med J 300: 1494-1497.

7 Stamey TA (1980) Endoscopic suspension of the vesical neck for urinary incontinence in females: report on 203 consecutive cases. Ann Surg 192: 465-471.

8 Stanton SL (1986) Colposuspension. In: Stanton SL, Tanagho EA, editors. Surgery of Female Incontinence. 2nd ed. Springer, Berlin. 